\title{
REFINED CONVERGENCE FOR THE BOOLEAN MODEL
}

\author{
PIERRE CALKA, ${ }^{*}$ Université Paris Descartes \\ JULIEN MICHEL, ${ }^{* *}$ ENS Lyon \\ KATY PAROUX, ${ }^{* * *}$ Université de Franche Comté and INRIA Rennes - Bretagne Atlantique
}

\begin{abstract}
In Michel and Paroux (2003) the authors proposed a new proof of a well-known convergence result for the scaled elementary connected vacant component in the high intensity Boolean model towards the Crofton cell of the Poisson hyperplane process (see, e.g. Hall (1985)). In this paper we investigate the second-order term in this convergence when the two-dimensional Boolean model and the Poisson line process are coupled on the same probability space. We consider the particular case where the grains are discs with random radii. A precise coupling between the Boolean model and the Poisson line process is first established. A result of directional convergence in distribution for the difference of the two sets involved is then derived. Eventually, we show the convergence of the process, measuring the difference between the two random sets, once rescaled, as a function of the direction.
\end{abstract}

Keywords: Poisson point process; Crofton cell; convergence; stochastic geometry

2000 Mathematics Subject Classification: Primary 60D05

Secondary 60G55; 60F99

\section{Introduction and notation}

Since it was first obtained by Hall [4], [5, p. 164] and generalised in [12] and [14], the convergence (in some sense) of the scaled vacancy of the Boolean model to its counterpart in the Poisson hyperplane process is well known. In a previous paper [9] two of the authors gave another proof of this convergence result for the local occupation laws of a Boolean shell model in terms of the Hausdorff distance. This convergence appears as a first-order result, expressed in terms of weak convergence. Our aim in this paper is to give two generalisations of this result. We first extend the weak convergence to an almost-sure convergence thanks to an adequate coupling between both models, and secondly we show a second-order weak convergence for the difference of both sets, expressed as the convergence of a stochastic process in the Skorokhod and $L^{1}$ senses.

We shall work in the plane $\mathbb{R}^{2}$, though some of our results might be stated in higher dimensions: let us consider a Boolean model based on a Poisson point process $\boldsymbol{X}_{\lambda}$ with intensity measure $\lambda \mathrm{d} x$ and generic shape an open disc centred at 0 of random radius $R$ such that $\mathrm{E}[R]=1$

\footnotetext{
Received 15 April 2008; revision received 29 June 2009.

* Postal address: MAP5, UFR de Mathématiques et Informatique, Université Paris Descartes, 45 rue des Saints-Pères, 75270 Paris Cedex 06, France. Email address: pierre.calka@math-info.univ-paris5.fr

Research partially supported by the French ANR project 'mipomodim', grant number ANR-05-BLAN-0017.

** Postal address: Unité de Mathématiques Pures et Appliquées, UMR 5669, ENS Lyon, 46 allée d'Italie, F-69364 Lyon Cedex 07, France.

*** Postal address: Laboratoire de Mathématiques de Besançon, UMR 6623, F-25030 Besançon Cedex, France.
} 
and $\mathrm{E}\left[R^{2}\right]<+\infty$. The law of $R$ will be denoted by $\mu$, and we shall assume that there exists $R_{\star}>0$ such that $\mu\left(R_{\star},+\infty\right)=1$.

The choice of a random disc enables us to write simply the different couplings and computations presented below; generic, convex smooth shapes could probably be treated in the same way, up to technical details.

The occupied phase of the Boolean model is denoted by

$$
\mathcal{O}_{\lambda}=\bigcup_{x \in X_{\lambda}} B\left(x, R_{x}\right),
$$

where $B(x, r)$ denotes the disc of radius $r$ centred at $x$, and the radii $R_{x}$ for each $x \in \boldsymbol{X}_{\lambda}$ are independent and identically distributed, independent of $\boldsymbol{X}_{\lambda}$ [13, p. 115]. This process is supposed to leave the point 0 uncovered, which occurs with positive probability

$$
\mathrm{P}\left(0 \notin \mathcal{O}_{\lambda}\right)=\exp \left(-\pi \lambda \mathrm{E}\left[R^{2}\right]\right) .
$$

From now on, the Boolean model will be conditioned by this event.

Let $D_{0}^{\lambda}$ denote the (closed) connected component of $\mathbb{R}^{2} \backslash \mathcal{O}_{\lambda}$ containing 0 . The following asymptotic result for this process (see [4], [9], [12], and [14]) may be seen as a consequence of Steiner's formula [16, p. 197].

Theorem 1. Let $D^{\lambda}$ be the following compact set:

- $D^{\lambda}=\lambda D_{0}^{\lambda}$ whenever this set is bounded;

- $D^{\lambda}=K_{0}$, a given fixed compact set, otherwise.

When $\lambda$ tends to $\infty, D^{\lambda}$ converges in law towards the Crofton cell $\mathcal{C}$ of a Poisson line process with intensity measure $\mathrm{d} \rho \mathrm{d} \theta$.

In [4] the convergence was stated for random discs and the Hausdorff distance, whereas in [12] it was proved for generic shapes, using the hit or miss topology for random closed sets. The criterion developed in [14] gives the convergence for another general class of shapes, whereas in [9] the convergence is proved using a convergence result for random shells.

The Poisson line process with intensity measure $\mathrm{d} \rho \mathrm{d} \theta$ in $\mathbb{R}^{2}$ is defined as the set of (random) lines $D_{\rho, \theta}=\left\{(r, t) \in \mathbb{R}_{+} \times[0,2 \pi): r \cos (t-\theta)=\rho\right\}$, where $(\rho, \theta)$ are the points of a Poisson point process $\Phi$ in $\mathbb{R}_{+} \times[0,2 \pi)$ with intensity measure $\mathrm{d} \rho \mathrm{d} \theta$. The Crofton cell $\mathcal{C}$ is defined as the polygon formed by those lines containing the point 0 (see [17, Foreword to the first edition] or [14] for a survey on Poisson line tessellations). Numerous distributional results on this model have been obtained, notably by Miles [10], [11] and Matheron [8]. More recently, central limit theorems have been derived in [14] and [15] for the two-dimensional case and in [6] for the general case. Besides, Kendall's conjecture on the shape of the Crofton cell when it is large has been proved in [7]. Additional distributional and asymptotic results at large inner radius have also been obtained in [2] and [3].

We shall first recall in Section 2 the asymptotic properties of the outer radius of the Crofton cell and give some counterpart of these asymptotics for the rescaled outer radius of the Boolean empty connected component. These results are useful for Sections 3, 4, and 5, and also give some insight into the behaviour of the high-intensity Boolean model with respect to the continuous percolation problem. It is a natural problem to try to estimate the error in Theorem 1: one possible answer is to give a geometric description of the difference of the two sets. This description requires coupling the Boolean model with the Poisson line process. This coupling, 
which asserts as a consequence the almost-sure convergence in Theorem 1, will be described in Section 3, and its application to the second-order convergence will be treated in Section 4 for directional convergence, and in Section 5 for the convergence of the rescaled defect process in the Skorokhod and $L^{1}$ settings.

\section{Estimates on the tail probability of the inner and outer radii}

This section contains autonomous results about the inner and outer radii of both the Crofton cell and the empty connected component $\lambda D_{0}^{\lambda}$. Let us recall their definitions and some elementary properties: the inner radius of the Crofton cell is defined by

$$
R_{m}=\sup \left\{r>0: B_{2}(0, r) \subset \mathcal{C}\right\}
$$

and the inner radius of the rescaled vacancy of the Boolean model is defined by

$$
R_{m}(\lambda)=\sup \left\{r>0: B_{2}(0, r) \subset \lambda D_{0}^{\lambda}\right\}
$$

It is clear that, for all $r>0$,

$$
\mathrm{P}\left(R_{m}>r\right)=\exp (-2 \pi r), \quad \mathrm{P}\left(R_{m}(\lambda)>r\right)=\exp \left(-\left(2 \pi r+\frac{\pi r^{2}}{\lambda}\right)\right) .
$$

The outer radius of the Crofton cell is defined by

$$
R_{M}=\inf \left\{r>0: \mathcal{C} \subset B_{2}(0, r)\right\},
$$

and the outer radius of the rescaled vacancy of the Boolean model is defined by

$$
R_{M}(\lambda)=\inf \left\{r>0: \lambda D_{0}^{\lambda} \subset B_{2}(0, r)\right\} .
$$

For the outer radius of the Crofton cell, we only have the following asymptotic result, proved in [2] in the context of a study of Kendall's conjecture on the shape of large Poisson polygons.

Theorem 2. (Theorem 8 of [2].) We have, for all $r>0$,

$$
\begin{aligned}
2 \pi \mathrm{e}^{-2 r} & \left(\cos 1+\frac{\mathrm{e}^{-(2 \pi \cos 1-1) r}}{2 \pi r}\right) \\
& \leq \mathrm{P}\left(R_{M} \geq r\right) \\
& \leq 2 \pi r \mathrm{e}^{-2 r}\left(1-(\pi-2) r \mathrm{e}^{-2 r}+\frac{2}{3}(\pi-3)^{2} r^{2} \mathrm{e}^{-4 r}+\frac{\mathrm{e}^{-2(\pi-1) r}}{2 \pi r}\right) .
\end{aligned}
$$

Concerning the Boolean model, we prove below the following counterpart.

Theorem 3. There exist a constant $S>0$ and constants $K$ and $C$ depending only on $R_{\star}$ such that, for all $r \geq 1$ and $\lambda \geq S r$, we have

$$
\mathrm{P}\left(R_{M}(\lambda)>r\right) \leq K \exp (-C r) .
$$

Proof. The proof of this theorem relies on the following (nonoptimal) reasoning. Let $N$ be a positive integer greater than 12 , and define the angular sectors $S_{i, N}$ for $i \in\{0, \ldots, N-1\}$ as

$$
S_{i, N}=\left\{(\rho, \theta): \rho>0, \theta \in\left[\frac{(2 i-1) \pi}{N}, \frac{(2 i+1) \pi}{N}\right)\right\} \text { for } i \in\{0, \ldots, N-1\} .
$$


If $\lambda D_{0}^{\lambda}$ is not included in $B(0, r)$ then there exists an $i$ such that no disc of the rescaled Boolean model contains both points with polar coordinates $(r,(2 i-1) \pi / N)$ and $(r,(2 i+1) \pi / N)$ (this implies that $\lambda$ must satisfy the condition $\lambda R \geq r \sin \pi / N)$.

Let us denote by $A_{\lambda, r, R}$ the set of the centres $(\rho, \theta)$ of discs of radius $\lambda R$ which contain $(r,-\pi / N)$ and $(r, \pi / N)$. We have, by invariance under rotations,

$$
\mathrm{P}\left(R_{M}(\lambda) \geq r\right) \leq N \exp \left(-\lambda^{-1} \int_{\mathbb{R}_{+} \times[0,2 \pi) \times \mathbb{R}_{+}} \mathbf{1}_{A_{\lambda, r, R}}(\rho, \theta) \rho \mathrm{d} \rho \mathrm{d} \theta \mathrm{d} \mu(R)\right),
$$

and the aim of the computations is to bound the Lebesgue measure $a_{\lambda, r, R}$ of the set $A_{\lambda, r, R}$ from below. This set is

$$
A_{\lambda, r, R}=\left[B\left(\left(r, \frac{\pi}{N}\right), \lambda R\right) \cap B\left(\left(r,-\frac{\pi}{N}\right), \lambda R\right)\right] \backslash B(0, \lambda R) .
$$

The geometry of this set is quite easily described. Indeed, let us introduce the angle $\theta_{0}=$ $\arccos (r / 2 \lambda R)-\pi / N$. Then, for $\lambda$ such that

$$
\lambda R \geq \max \left(\frac{r}{2 \cos \left(\theta_{0}-\pi / N\right)}, \frac{r}{2 \cos (\pi / N)}\right),
$$

we have

$$
(\rho, \theta) \in A_{\lambda, r, R} \quad \text { if and only if } \quad-\theta_{0} \leq \theta \leq \theta_{0} \text { and } \lambda R \leq \rho \leq \rho_{e}(\theta),
$$

where $\rho_{e}(\theta)$ is given by

$$
\rho_{e}(\theta)=r \cos \left(|\theta|+\frac{\pi}{N}\right)+\lambda R \sqrt{1-\left(\frac{r \sin (|\theta|+\pi / N)}{\lambda R}\right)^{2}} .
$$

Let us now introduce $\theta_{1}=\pi / 12$. This angle satisfies $\theta_{1}<\theta_{0}$ for large enough $\lambda(\lambda \geq S r$, where the constant $S$ is chosen greater than 2, and depends on $N$ and $\left.R_{\star}\right)$. The computation of $a_{\lambda, r, R}$ becomes

$$
\begin{aligned}
a_{\lambda, r, R}= & \int_{0}^{\theta_{0}}\left[\rho_{e}(\theta)^{2}-(\lambda R)^{2}\right] \mathrm{d} \theta, \\
\geq & \int_{0}^{\theta_{1}}\left[\rho_{e}(\theta)^{2}-(\lambda R)^{2}\right] \mathrm{d} \theta, \\
\geq & \int_{0}^{\theta_{1}}[\underbrace{r^{2} \cos (2 \theta+2 \pi / N)}_{\geq 0} \\
& \quad+2 r \cos \left(\theta+\frac{\pi}{N}\right) \underbrace{\sqrt{\lambda^{2} R^{2}-r^{2} \sin ^{2}(\theta+\pi / N)}}_{\geq(\sqrt{3} / 2) \lambda R \text { from }(2)}] \mathrm{d} \theta, \\
\geq & \frac{3 \pi}{24} \lambda R r .
\end{aligned}
$$

Inserting this estimate into inequality (1) completes the proof of Theorem 3. 


\section{Coupling and almost-sure convergence}

Coupling the Boolean model with the Poisson line process is an easy task; indeed, as $\lambda$ tends to $\infty$, the rescaled Boolean model looks like the Poisson line process, as can be seen from Theorem 1 of [9]. The formal way to state this as a coupling result is to introduce a marked Poisson line process which couples both processes. Let $\boldsymbol{Z}$ be a Poisson point process with intensity measure $\mathrm{d} \rho \mathrm{d} \theta \mathrm{d} \mu$ on $\mathbb{R}_{+} \times[0,2 \pi) \times \mathbb{R}_{+}$, and define the function

$$
\psi_{\lambda}(\rho, R)=R \sqrt{1+\frac{2 \rho}{\lambda R}} .
$$

Define the following processes:

- $\boldsymbol{X}=\bigcup_{(\rho, \theta, R) \in \boldsymbol{Z}}\{(\rho, \theta)\}$;

- $\boldsymbol{X}_{\lambda}^{M}=\bigcup_{(\rho, \theta, R) \in \boldsymbol{Z}}\left\{\left(\psi_{\lambda}(\rho, R), \theta, R\right)\right\}$.

Then we have the following proposition.

Proposition 1. The processes $\boldsymbol{X}$ and $\boldsymbol{X}_{\lambda}^{M}$ are Poisson point processes with respective intensities $\mathrm{d} \rho \mathrm{d} \theta$ and $\lambda \rho \mathrm{d} \rho \mathrm{d} \theta \mathrm{d} \mu$ on $\mathbb{R}_{+} \times[0,2 \pi)$ and $\mathcal{B}:=\left\{(r, t, R) \in \mathbb{R}_{+} \times[0,2 \pi) \times \mathbb{R}_{+}: r>R\right\}$, respectively.

This proposition gives the coupling between the Boolean model and the Poisson line process in the following way: on the one hand, we construct the Boolean model associated to the marked points of the process $\boldsymbol{X}_{\lambda}^{M}$, and on the other hand, we construct the polar lines at the points of $\boldsymbol{X}$. If we rescale the Boolean model by a factor $\lambda$, we obtain the picture presented in Figure 1.

Remark 1. Conversely, we could have introduced the coupling starting from the points $(\rho, \theta, R)$ of a marked Poisson point process $\boldsymbol{X}_{\lambda}^{M}$ with intensity $\lambda \rho \mathrm{d} \rho \mathrm{d} \theta \mathrm{d} \mu$ on $\mathcal{B}$, yielding directly the Boolean model. In this setting the application

$$
(\rho, \theta, R) \mapsto\left(\lambda(\rho-R)+\frac{1}{2 R} \lambda(\rho-R)^{2}, \theta\right)
$$

maps $\boldsymbol{X}_{\lambda}^{M}$ onto a Poisson point process $\boldsymbol{X}$ with intensity measure $\mathrm{d} \rho \mathrm{d} \theta$ on which we may construct the Poisson line process.

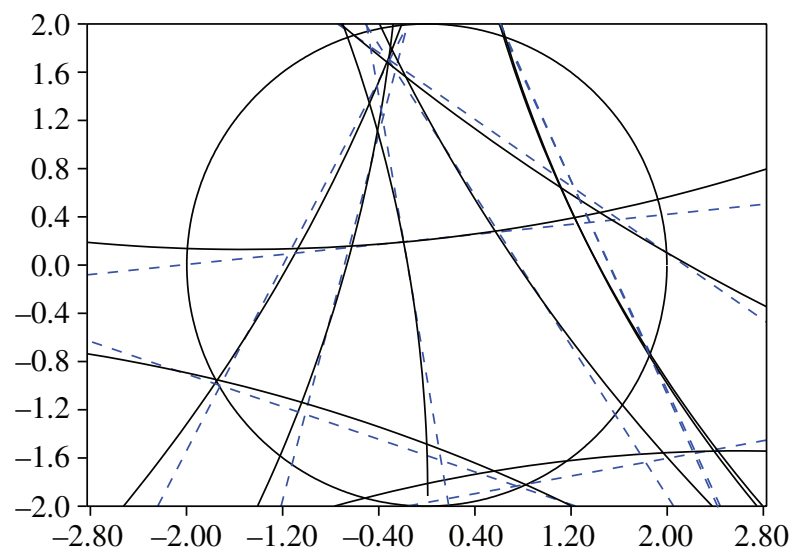

FIGURE 1: Coupling the Boolean model with the line process (the simulation is exact within the circle). 
From now on we shall use the coupling induced by $Z$; thus, the set $D^{\lambda}$ will refer to the rescaled connected component in this Boolean model (when bounded, and $K_{0}$ otherwise), and $\mathcal{C}$ will be the Crofton cell in this line process.

This coupling yields the following result on the local accuracy of the approximation of the rescaled Boolean model by the line process.

Proposition 2. For all $M>0$ and $\lambda \geq \lambda_{0}(M)=M / R_{\star}>0$, we have

$$
d_{H}^{M}\left(\lambda D_{0}^{\lambda}, \mathcal{C}\right) \leq \frac{M^{\prime 2}}{R_{\star} \lambda} \quad \text { almost surely, }
$$

where $M^{\prime}=M+M^{2} /\left(\lambda R_{\star}\right), d_{H}^{M}$ denotes the M-Hausdorff distance in $B(0, M)$ defined by

$$
\begin{aligned}
& d_{H}^{M}(F, G)=\inf \{\alpha>0:(F \oplus B(0, \alpha)) \cap B(0, M) \supset G \cap B(0, M), \\
& (G \oplus B(0, \alpha)) \cap B(0, M) \supset F \cap B(0, M)\},
\end{aligned}
$$

and, for any two subsets $A$ and $B$, the set $A \oplus B$ denotes their Minkowski sum: $A \oplus B=$ $\{x+y,(x, y) \in A \times B\}$.

Proof. Let us denote by $\left(\rho_{1}, \alpha_{1}, R_{1}\right), \ldots,\left(\rho_{K}, \alpha_{K}, R_{K}\right)$ the points of $\boldsymbol{Z}$ such that the rescaled discs $B_{i}$ associated to these points intersect $B(0, M)$. Since $\lambda \geq \lambda_{0}(M)$, no such disc can be included in $B(0, M)$. A straightforward computation with the help of (3) shows that the associated lines intersect the disc $B\left(0, M^{\prime}\right)$, where

$$
M^{\prime}=M+\frac{M^{2}}{\lambda R_{\star}} .
$$

More precisely, as is shown in Figure 2, the $M$-Hausdorff distance between the intersection of the circle $\partial B_{i}$ with $B(0, M)$ and the intersection of the tangent line $T_{i}$ with $B(0, M)$ is bounded by the distance between the points $A$ and $C$, defined respectively as the intersection of $T_{i}$ and

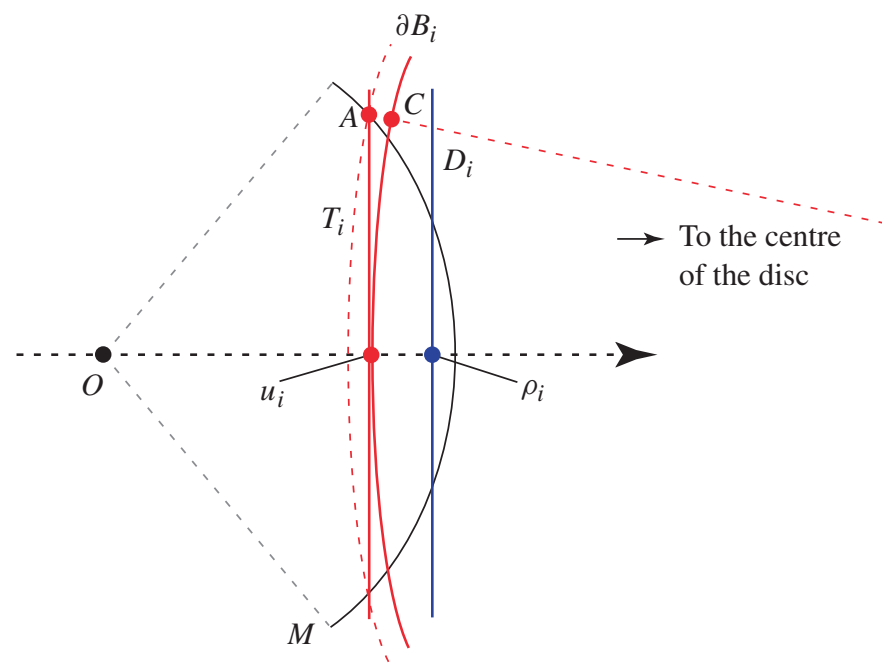

FIGURE 2: The circle $\partial B_{i}$ and the tangent line $T_{i}$. 
$\partial B(0, M)$, and the point on $\partial B_{i}$ aligned with $A$ and the centre of $B_{i}$. We have, if we define the distance from the origin to $B_{i}$ as $u_{i}=\lambda R_{i}\left(\sqrt{1+2 \rho_{i} /\left(\lambda R_{i}\right)}-1\right) \leq M$,

$$
A C=\sqrt{\lambda^{2} R_{i}^{2}+M^{2}-u_{i}^{2}}-\lambda R_{i}=\frac{M^{2}-u_{i}^{2}}{\sqrt{\lambda^{2} R_{i}^{2}+M^{2}-u_{i}^{2}}+\lambda R_{i}} \leq \frac{M^{2}}{2 \lambda R_{i}} .
$$

Consequently, for any disc $B$ of radius $\lambda R>\lambda R_{\star}$ such that $0 \notin B$, and $T$ the tangent to this disc at its closest point to 0 , we have

$$
d_{H}^{M}(\partial B, T) \leq \frac{M^{2}}{2 \lambda R_{\star}} .
$$

On the other hand, as $u_{i} \leq M$, we have

$$
\left|u_{i}-\rho_{i}\right|=\rho_{i}\left|\frac{1-\sqrt{1+2 \rho_{i} / \lambda R_{i}}}{1+\sqrt{1+2 \rho_{i} / \lambda R_{i}}}\right| \leq \frac{\rho_{i}^{2}}{2 \lambda R_{i}} \leq \frac{M^{\prime 2}}{2 \lambda R_{\star}},
$$

so that we obtain the result of Proposition 2 by combining the two inequalities in (4) and (5): if we denote by $H_{i}$ and $H_{i}^{\prime}$ the half-planes with boundary $D_{i}$ and boundary $T_{i}$, respectively, not containing the origin,

$$
\begin{aligned}
d_{H}^{M}\left(\lambda D_{0}^{\lambda}, C_{0}^{\lambda}\right) & \leq \max _{i \in\{1, \ldots, K\}} d_{H}^{M}\left({ }^{\mathrm{c}} B_{i},{ }^{\mathrm{c}} H_{i}\right), \\
& \leq \max _{i \in\{1, \ldots, K\}} d_{H}^{M}\left({ }^{\mathrm{c}} B_{i},{ }^{\mathrm{c}} H_{i}^{\prime}\right)+\max _{i \in\{1, \ldots, K\}} d_{H}^{M}\left({ }^{\mathrm{c}} H_{i},{ }^{\mathrm{c}} H_{i}^{\prime}\right), \\
& \leq \frac{M^{\prime 2}}{\lambda R_{\star}},
\end{aligned}
$$

where ${ }^{\mathrm{c}} G$ denotes the complementary set of $G$. This concludes the proof.

From Proposition 2 we may deduce the almost-sure convergence in our coupled setting.

Theorem 4. Almost surely, $D^{\lambda}$ converges in Hausdorff distance towards $\mathcal{C}$.

Proof. Let us consider the subset $\Omega_{r}$ of those $\omega$ s for which both $R_{M}$ and $R_{M}(\lambda)$ are less than $r$. Then the Hausdorff distance between $\mathcal{C}$ and $D^{\lambda}=\lambda d_{0}^{\lambda}$ is less than $r^{\prime 2} /\left(R_{\star} \lambda\right)$, where $r^{\prime}=r+r^{2} /\left(\lambda R_{\star}\right)$ for large enough $\lambda$ thanks to Proposition 2, and $\mathrm{P}\left(\Omega_{r}\right) \rightarrow 1$ as $r \rightarrow+\infty$ thanks to Theorems 2 and 3. This completes the proof.

Remark 2. The actual speed of convergence shown above could be stated in the following way. Let $S_{M}(\lambda)=\max \left(R_{M}, R_{M}(\lambda)\right)$. Then

$$
d_{H}\left(\mathcal{C}, D^{\lambda}\right) \leq \frac{S_{M}^{\prime}(\lambda)^{2}}{\lambda R_{\star}},
$$

where $S_{M}^{\prime}(\lambda)=S_{M}(\lambda)+S_{M}(\lambda)^{2} /\left(\lambda R_{\star}\right)$.

\section{Convergence of the second-order directional results}

In order to prove a second-order convergence result for the empty connected component towards the Crofton cell, we shall first give some notation and definitions, then we shall state the convergence results for one, then many directions in Sections 4.2 and 4.3. 


\subsection{Notation}

Recall that, for each $\omega \in \Omega$, we denote by $\mathcal{C}(\omega)$ the Crofton cell of the Poisson line process $\boldsymbol{X}$ induced by $\boldsymbol{Z}$, and by $D^{\lambda}(\omega)$ the empty connected component of the rescaled coupled Boolean model. We define the following quantities (almost surely, they are all finite random variables):

- $N_{e}(\omega)$ is the number of vertices of $\mathcal{C}(\omega)$, which are denoted anticlockwise by $V_{1}(\omega), \ldots$, $V_{N_{e}(\omega)}(\omega)$;

- $0 \leq \theta_{1}(\omega)<\cdots<\theta_{N_{e}(\omega)}(\omega)<2 \pi$ are the polar angles of the vertices of $\mathcal{C}(\omega)$;

- we take the convention that an edge of $\mathcal{C}(\omega)$ numbered $i$ joins vertices $V_{i}$ (included) and $V_{\left(i \bmod N_{e}(\omega)\right)+1}($ excluded);

- for each $i \in\left\{1, \ldots, N_{e}(\omega)\right\}$, set $\left(\Upsilon_{i}(\omega), \Theta_{i}(\omega), R_{i}(\omega)\right)$ to be the polar coordinates (angle and distance) of the edges of $\mathcal{C}(\omega)$ marked with the associated radius of the disc in the coupled Boolean model (from now on we shall write $i+1$ for $\left(i \bmod N_{e}(\omega)\right)+1$ and $N_{e}$ for $N_{e}(\omega)$ for simplicity).

For each $\omega \in \Omega$ and $t \in[0,2 \pi)$, we define $\Delta_{t}$ to be the half-line $\{(r, t): r>0\}$ and

- $\Theta(t, \omega)$ to be the polar angle of the edge intersecting the half-line $\Delta_{t}$;

- $\Upsilon(t, \omega)$ to be the distance from the origin to this edge;

- $L(t, \omega)=\Upsilon(t, \omega) / \cos (\Theta(t, \omega)-t)$ to be the distance from the origin to the intersection of $\Delta_{t}$ with this edge;

- $R(t, \omega)$ to be the radius of the associated disc.

All the quantities above are well defined on the same set of full probability for each $t$.

Definition 1. For each $\omega \in \Omega$ and $t \in[0,2 \pi)$, we define the defect at angle $t$ by

$$
d_{\lambda}(t, \omega)=\operatorname{dist}\left(0, \Delta_{t} \cap \partial D^{\lambda}(\omega)\right)-\operatorname{dist}\left(0, \Delta_{t} \cap \partial \mathcal{C}(\omega)\right),
$$

where dist denotes the Euclidean distance, and the approximate defect at angle $t$ by

$$
\bar{d}_{\lambda}(t, \omega)=\operatorname{dist}\left(0, \Delta_{t} \cap B\left(\lambda \psi_{\lambda}(\Upsilon(t, \omega), \Theta(t, \omega)), \lambda R(t, \omega)\right)\right)-\operatorname{dist}\left(0, \Delta_{t} \cap \mathcal{C}(\omega)\right),
$$

when this quantity is well defined (for large enough $\lambda$ ).

We can easily check that

$$
\begin{aligned}
\bar{d}_{\lambda}(t, \omega)= & \lambda R(t, \omega) \cos (\Theta(t, \omega)-t) \sqrt{1+\frac{2 \Upsilon(t, \omega)}{\lambda R(t, \omega)}} \\
& -\lambda R(t, \omega) \sqrt{1-\sin ^{2}(\Theta(t, \omega)-t)\left(1+\frac{2 \Upsilon(t, \omega)}{\lambda R(t, \omega)}\right)} \\
& -\frac{\Upsilon(t, \omega)}{\cos (\Theta(t, \omega)-t)}
\end{aligned}
$$

for $\lambda \geq 2 \Upsilon(t, \omega) \tan ^{2}(\Theta(t, \omega)-t) / R(t, \omega)$; see Figure 3 . 


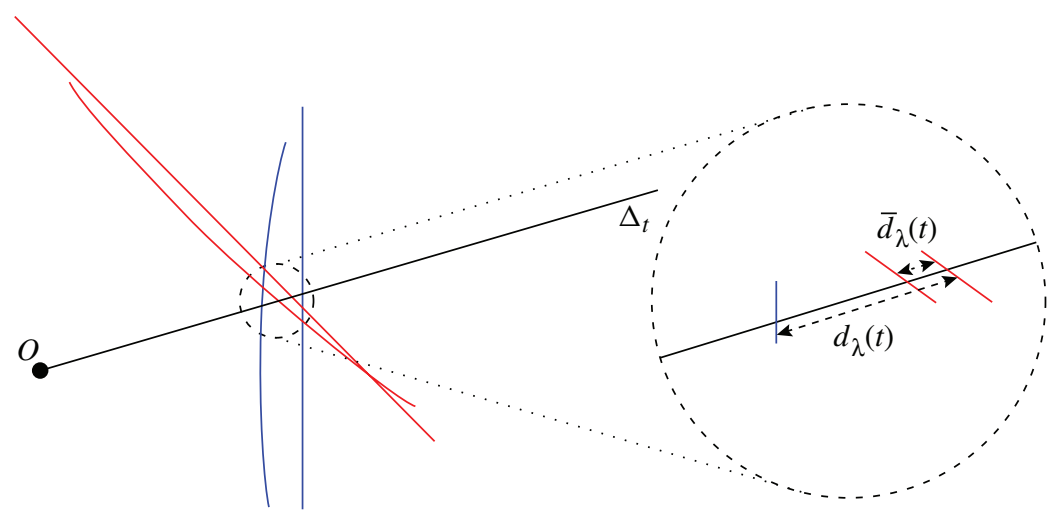

FIGURE 3: Defect and approximate defect.

\subsection{One directional convergence}

The first result is an almost-sure convergence (denoted by ' $\stackrel{\text { a.s. }}{\longrightarrow}$ ) of the defect function in one fixed direction.

Theorem 5. For all $t \in[0,2 \pi)$, we have

$$
\lambda d_{\lambda}(t, \cdot) \stackrel{\text { a.s. }}{\longrightarrow} Z(t, \cdot) \text { as } \lambda \rightarrow+\infty,
$$

where $Z(t, \cdot)$ is the random variable defined by

$$
Z(t, \omega)=-\frac{L(t, \omega)^{2}}{2} \frac{\cos 2(\Theta(t, \omega)-t)}{R(t, \omega) \cos (\Theta(t, \omega)-t)} \quad \text { for all } \omega \in \Omega,
$$

and the common law of $(L(t, \cdot), \Theta(t, \cdot), R(t, \cdot))$ is given by

$$
\begin{aligned}
& \mathrm{d}(L(t, \cdot), \Theta(t, \cdot), R(t, \cdot))(P)(\ell, \alpha, r) \\
& \quad=\pi \exp (-2 \pi \ell) \cos (\alpha-t) \mathbf{1}_{\{\alpha \in(t-\pi / 2, t+\pi / 2)\}} \mathrm{d} \ell \mathrm{d} \alpha \mathrm{d} \mu(r) .
\end{aligned}
$$

Proof. The proof of this result proceeds in two steps.

Step 1. Restrict the probability space to events in which, for large enough $\lambda$, the defect is equal to the approximate defect.

Step 2. Show that these events cover $\Omega$ almost surely.

Step 1: Restricted events. Let us consider $\delta>0, \varepsilon>0, r>0$, and $s>0$, and the subset $\Omega_{\delta, \varepsilon, r, s}$ of all $\omega \in \Omega$ such that

- $B(0, r) \subset \mathcal{C}(\omega) \subset B(0, s)$;

- for each $u \in(t-\delta, t+\delta)$, the intersection of $\mathcal{C}(\omega)$ with $\Delta_{u}$ is on the same edge of $\mathcal{C}(\omega)$;

- $\mathcal{C}(\omega) \oplus B(0, \varepsilon)$ is not intersected by lines of the Poisson line process, other than those on the boundary of $\mathcal{C}(\omega)$. 
It is quite obvious for geometrical arguments that, if $\lambda$ is large enough, in direction $t$ the defect will be exactly equal to the approximate defect, as in the disc of radius $s+\varepsilon$ the Hausdorff distance between circles and lines gets smaller as $\lambda$ increases, and, thus, in direction $t$ the first intersecting line corresponds to the first intersecting disc. It remains to compute the exact asymptotics of the approximate defect, which is done in the following way, where we restrict ourselves, thanks to invariance under rotations, to the angle $t=0$.

On the one hand, we have the following well-known classical result for the law of the first intersecting line.

Lemma 1. Let $L$ denote the distance from 0 to the first intersection on $\Delta_{0}$ of the line process, and let $\Theta$ denote the polar angle of this intersecting line. Then the law of $(L, \Theta)$ is given by

$$
\mathrm{d}(L, \Theta)(P)(\ell, \theta)=\mathrm{e}^{-2 \ell} \cos \theta \mathbf{1}_{\{\theta \in(-\pi / 2, \pi / 2)\}} \mathrm{d} \ell \mathrm{d} \theta .
$$

On the other hand, from (6) we easily obtain

$$
\begin{aligned}
d_{\lambda}(0)= & \bar{d}_{\lambda}(0) \\
= & \lambda R(0, \omega) \cos \Theta(0, \omega) \sqrt{1+\frac{2 L(0, \omega) \cos \Theta(0, \omega)}{\lambda R(0, \omega)}} \\
& -\lambda R(0, \omega) \sqrt{1-\sin ^{2} \Theta(0, \omega)\left(1+\frac{2 L(0, \omega) \cos \Theta(0, \omega)}{\lambda R(0, \omega)}\right)}-L(0, \omega)
\end{aligned}
$$

if the inner term of the square root is nonnegative (i.e. $\lambda \geq\left(2 L \sin ^{2} \Theta\right) /(R \cos \Theta)$ ), and $+\infty$ otherwise. The asymptotic expansion of the square roots easily gives

$$
\bar{d}_{\lambda}(0)=-\frac{L(0, \omega)^{2}}{2 \lambda} \frac{\cos 2 \Theta(0, \omega)}{R(0, \omega) \cos \Theta(0, \omega)}+O\left(\lambda^{-1}\right) .
$$

Step 2: Almost-sure covering. We conclude the proof of Theorem 5 by stating the following lemma.

Lemma 2. As $\delta, r$, and $\varepsilon$ tend to 0 and s tends to $+\infty$, we have $\mathrm{P}\left(\Omega_{\delta, \varepsilon, r, s}\right) \rightarrow 1$.

The proof of this lemma comes directly from the properties of the Poisson point process $\boldsymbol{X}$ and the asymptotic results on the law of the inner and outer radii of the Crofton cell stated in Section 2.

Remark 3. The almost-sure convergence above will not be used for the convergence of the defect process, only the convergence in law of the finite directional distributions is needed; however, we shall state them almost surely.

\subsection{Two and more directions}

For more directions, we may state similar results.

Theorem 6. For all $0 \leq t_{1}<\cdots<t_{n}<2 \pi$, the finite-dimensional random vector $\lambda\left(d_{\lambda}\left(t_{1}, \cdot\right)\right.$, $\left.\ldots, d_{\lambda}\left(t_{n}, \cdot\right)\right)$ converges almost surely towards $\left(Z\left(t_{1}, \cdot\right), \ldots, Z\left(t_{n}, \cdot\right)\right)$, where the law of this random vector may be made explicit.

The proof is essentially the same as for one direction, only with more technical details.

This random vector depends only on the characteristics of the Crofton cell. Let us, e.g. give the exact law of this vector for two directions (by invariance under rotations we choose directions 0 and $t \in(0,2 \pi))$. The random vector $\lambda\left(d_{\lambda}(0, \cdot), d_{\lambda}(t, \cdot)\right)$ converges in law as $\lambda$ goes 
to $\infty$ towards $T\left(Z_{0}(\Upsilon, \Theta, R), Z_{t}(\Upsilon, \Theta, R)\right)+(1-T)\left(W_{0}\left(\Upsilon_{1}, \Theta_{1}, R_{1}\right), W_{t}\left(\Upsilon_{2}, \Theta_{2}, R_{2}\right)\right)$, where

- $T$ is a Bernoulli random variable stating that the same line determines the intersections in directions 0 and $t$ : this occurs with probability $p$,

$$
\begin{aligned}
p & =\mathrm{E}\left[\sum_{(\rho, \alpha, R) \in \boldsymbol{Z}} \mathbf{1}_{\left.\left\{\text {The lines from } \boldsymbol{Z} \backslash\{\rho, \alpha, R\} \text { do not intersect } \Delta_{0} \text { or } \Delta_{t} \text { before } D_{(\rho, \alpha)}\right\}\right],}\right. \\
& =\int_{\mathbb{R}_{+} \times[0,2 \pi) \times\left[R_{\star},+\infty\right)} \exp \left(-\mathfrak{p}\left(\Delta_{0, t}(\rho, \alpha)\right)\right) \mathrm{d} \rho \mathrm{d} \alpha \mathrm{d} \mu(r),
\end{aligned}
$$

where $\Delta_{0, t}(\rho, \alpha)$ is the triangle described by Figure 4 and $\mathfrak{p}$ denotes the perimeter function; this Bernoulli random variable is independent from the following ones;

- $(\Upsilon, \Theta, R)$ has the following distribution:

$$
\begin{aligned}
\mathrm{d}(\Upsilon, \Theta, R)(P)(\rho, \alpha, r)= & p^{-1} \mathbf{1}_{\{\alpha \in(-\pi / 2, \pi / 2), \alpha-t \in(-\pi / 2, \pi / 2)\}} \\
& \times \exp \left(-\mathfrak{p}\left(\Delta_{0, t}(\rho, \alpha)\right)\right) \mathrm{d} \rho \mathrm{d} \alpha \mathrm{d} \mu(r) ;
\end{aligned}
$$

- $Z_{0}(\Upsilon, \Theta, R)=-\frac{\Upsilon^{2}}{2 R \cos ^{2} \Theta} \frac{\cos 2 \Theta}{\cos \Theta}$;

- $Z_{t}(\Upsilon, \Theta, R)=-\frac{\Upsilon^{2}}{2 R \cos ^{2}(\Theta-\theta)} \frac{\cos 2(\Theta-t)}{\cos (\Theta-t)}$;

- $\left(\Upsilon_{1}, \Theta_{1}, R_{1}, \Upsilon_{2}, \Theta_{2}, R_{2}\right)$ has the following distribution:

$$
\begin{aligned}
\mathrm{d}\left(\Upsilon_{1}, \Theta_{1}, R_{1}, \Upsilon_{2}, \Theta_{2}, R_{2}\right)(P)\left(\rho_{1}, \alpha_{1}, r_{1}, \rho_{2}, \alpha_{2}, r_{2}\right) \\
=(1-p)^{-1} \mathbf{1}_{\left\{\alpha_{1} \in(-\pi / 2, \pi / 2)\right\}} \mathbf{1}_{\left\{\alpha_{2}-t \in(-\pi / 2, \pi / 2)\right\}} \mathbf{1}_{\left\{\left(\rho_{1}, \alpha_{1}\right) \notin B_{t}\left(\rho_{2}, \alpha_{2}\right)\right\}} \mathbf{1}_{\left\{\left(\rho_{2}, \alpha_{2}\right) \notin B_{0}\left(\rho_{1}, \alpha_{1}\right)\right\}} \\
\quad \times \exp \left(-\mathfrak{p}\left(\Delta_{0, t}^{\prime}\left(\rho_{1}, \alpha_{1}, \rho_{2}, \alpha_{2}\right)\right)\right) \mathrm{d} \rho_{1} \mathrm{~d} \alpha_{1} \mathrm{~d} \mu\left(r_{1}\right) \mathrm{d} \rho_{2} \mathrm{~d} \alpha_{2} \mathrm{~d} \mu\left(r_{2}\right),
\end{aligned}
$$

where the sets $B_{0}, B_{t}$, and $\Delta_{0, t}^{\prime}$ are described by Figure 4;

- $W_{0}\left(\Upsilon_{1}, \Theta_{1}, R_{1}\right)=-\frac{\Upsilon_{1}^{2}}{2 R_{1} \cos ^{2} \Theta_{1}} \frac{\cos 2 \Theta_{1}}{\cos \Theta_{1}}$;

- $W_{\theta}\left(\Upsilon_{2}, \Theta_{2}, R_{2}\right)=-\frac{\Upsilon_{2}^{2}}{2 R_{2} \cos ^{2}\left(\Theta_{2}-t\right)} \frac{\cos 2\left(\Theta_{2}-t\right)}{\cos \left(\Theta_{2}-t\right)}$.

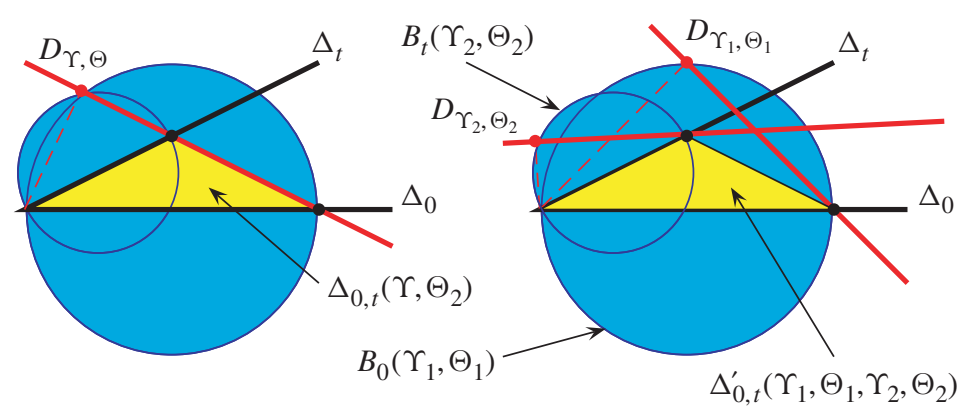

FIGURE 4: The sets $\Delta_{0, t}, \Delta_{0, t}^{\prime}, B_{0}$, and $B_{t}$, and the corresponding lines. 


\section{Convergence of the stochastic process}

In this section we consider the processes $\left(\lambda d_{\lambda}(t)\right)_{t \in[0,2 \pi)}$. Let us first note that knowing the joint limit law of the couples $\left(\lambda d_{\lambda}(0), \lambda d_{\lambda}(t)\right)$ gives some knowledge on this process; for instance, by simulation we can obtain the covariogram $t \mapsto \operatorname{cov}\left(\lambda d_{\lambda}(0), \lambda d_{\lambda}(t)\right), t \in[0, \pi]$, in Figure 5. We clearly observe the divergence as $\lambda$ tends to $\infty$ of the covariance for $t \rightarrow 0$; this is a consequence of the following elementary result coming from the explicit law of the defect.

Corollary 1. The limit expected defect is an integrable random variable with

$$
\lim _{\lambda \rightarrow+\infty} \mathrm{E}\left[\lambda d_{\lambda}(0)\right]=0 .
$$

However, this limit expected defect is not square integrable:

$$
\lim _{\lambda \rightarrow+\infty} \mathrm{E}\left[\left(\lambda d_{\lambda}(0)\right)^{2}\right]=+\infty
$$

The matter of convergence of the whole process will be stated in the space $D([0,2 \pi])$. As a matter of fact, for each $\lambda$, the trajectory of the defect process is continuous; however, the limit process is not continuous: the choice of the space $D([0,2 \pi])$ of right-continuous functions having a limit to the left (càdlàg functions) on $[0,2 \pi]$, even if there is no geometric justification in choosing right continuity, seems to be quite natural. Let us thus consider such processes $X_{\lambda}$ on $[0,2 \pi]$, according to Theorem 13.3 of $[1$, p. 141], the conditions for the convergence of processes $\left(X_{\lambda}\right)_{\lambda \geq 1}$ on $D([0,2 \pi])$ are

- convergence in law of the finite-dimensional distributions, which is true thanks to Theorem 6;

- the tightness criterion; for instance, for all $\eta, \varepsilon>0$, there exists $\delta>0$ such that

$$
\limsup _{\lambda \rightarrow+\infty} \mathrm{P}\left(\sup _{t_{1} \leq t \leq t_{2}, t_{2}-t_{1}<\delta} \min \left(\left|X_{\lambda}(t)-X_{\lambda}\left(t_{1}\right)\right|,\left|X_{\lambda}\left(t_{2}\right)-X_{\lambda}(t)\right|\right) \geq \varepsilon\right) \leq \eta .
$$

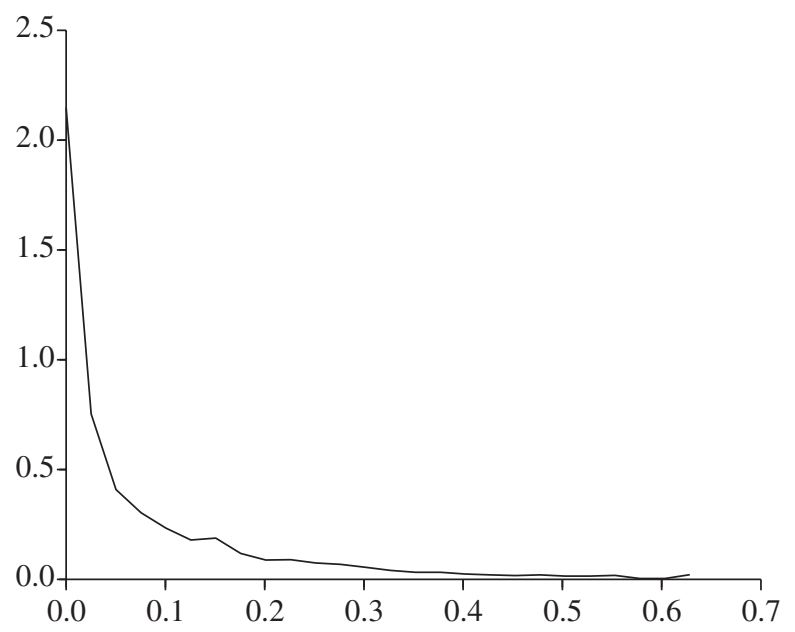

FIgURE 5: Covariogram for $\lambda=1000$ and a sample of size 250000. 

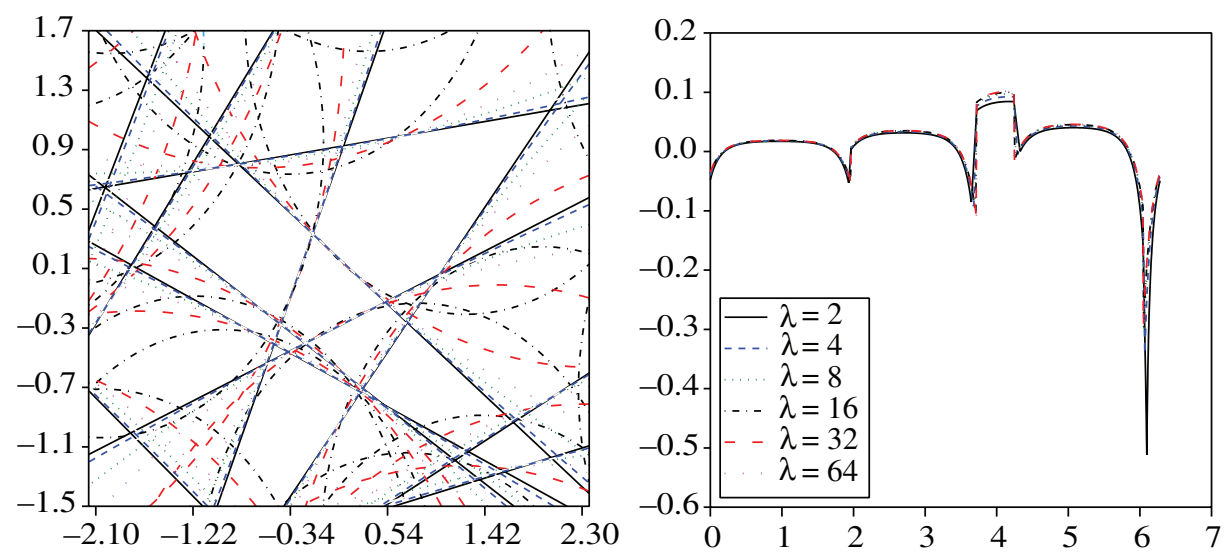

Figure 6: The Crofton cell and related processes. We check that there are four edges and four singularities.

In our case, unfortunately, we cannot use such a tightness criterion directly; indeed, if we take $X_{\lambda}=\lambda d_{\lambda}$, we see (Figure 6) that the high slopes that appear near the angles corresponding to the vertices of the Crofton cell forbid us to use this kind of criterion, as well as all other classical criteria. Hence, we shall first show the convergence of the approximate defect process $X_{\lambda}=\lambda \bar{d}_{\lambda}$, as this process is the combination of a jump process and a smooth process, and then give an explicit estimate on the accuracy of this approximation in the $L^{1}$-norm.

\subsection{Convergence of the approximate defect process}

We prove the following theorem on the approximate defect.

Theorem 7. The approximate defect process $\left(\lambda \bar{d}_{\lambda}(t)\right)_{t \in[0,2 \pi]}$ converges in law in $D([0,2 \pi])$ to the process $\left(X_{t}\right)_{t \in[0,2 \pi]}$ defined, for all $i \in\left\{1, \ldots, N_{e}\right\}$ and $t \in\left[\theta_{i}, \theta_{i+1}\right)$, by

$$
\omega \mapsto X_{t}(\omega)=-\frac{\Upsilon_{i}(t, \omega)^{2}}{2 R_{i}(t, \omega)} \frac{\cos 2\left(\Theta_{i}(t, \omega)-t\right)}{\cos ^{3}\left(\Theta_{i}(t, \omega)-t\right)},
$$

using the notation in Section 4.1.

Proof. Let us fix $\varepsilon$ and $\eta$, both positive numbers, and define, for $0<r<s$ and $\delta_{0}>0$, the set $\Omega_{r, R, \delta_{0}}$ of those $\omega \in \Omega$ such that the Crofton cell $\mathcal{C}(\omega)$ and $D^{\lambda}$ satisfy the following conditions:

- $B(0, r) \subset \mathcal{C}(\omega) \subset B(0, s)$, i.e. $R_{m} \geq r$ and $R_{M} \leq s$;

- $D^{\lambda} \subset B(0, s)$, i.e. $R_{M}(\lambda) \leq s$;

- the angular distance $\theta_{i+1}(\omega)-\theta_{i}(\omega)$ between any two consecutive vertices of $\mathcal{C}(\omega)$ is greater than $\delta_{0}$.

On the event $\Omega_{r, s, \delta_{0}}$, we can easily check from Definition 1 that, for $t \in\left[\theta_{i}, \theta_{i+1}\right)$, the approximate defect $\lambda \bar{d}_{\lambda}(t)$ is well defined for $\lambda \geq 2 s^{3} /\left(r^{2} R_{\star}\right)$ and is Lipschitz continuous for $\lambda \geq 4 s^{3} /\left(r^{2} R_{\star}\right)$ with Lipschitz constant less than $13 s^{6} /\left(r^{4} R_{\star}\right)$ on the interval $\left[\theta_{i}, \theta_{i+1}\right)$.

It is then straightforward to check that, if we choose $\delta<\delta_{0}$ and $\delta<C \varepsilon r^{4} R_{\star} / s^{6}$, we have, for large enough $\lambda$ (depending on $r$ and $s$ ),

$$
\mathrm{P}\left(\sup _{t_{1} \leq t \leq t_{2}, t_{2}-t_{1}<\delta} \min \left(\left|\lambda \bar{d}_{\lambda}(t)-\lambda \bar{d}_{\lambda}\left(t_{1}\right)\right|,\left|\lambda \bar{d}_{\lambda}\left(t_{2}\right)-\lambda \bar{d}_{\lambda}(t)\right|\right) \geq \varepsilon\right) \leq 1-\mathrm{P}\left(\Omega_{r, s, \delta_{0}}\right) .
$$


We then complete the proof of Theorem 7 using the following lemma, similar to Lemma 2, which states that, with high probability, the Crofton cell is a 'gentle' polygon.

Lemma 3. As $r \rightarrow 0, s \rightarrow+\infty$, and $\delta_{0} \rightarrow 0$, we have $\mathrm{P}\left(\Omega_{r, s, \delta_{0}}\right) \rightarrow 1$.

The asymptotic expansion of the approximate defect also gives a convergence in the spaces $L^{p}(0,2 \pi), p \in[1,+\infty]$, of measurable functions $f$ defined on $(0,2 \pi)$ such that

$\int_{(0,2 \pi)}|f(t)|^{p} \mathrm{~d} t<+\infty \quad$ for $p<+\infty, \quad$ or $\quad \operatorname{ess} \sup _{t \in(0,2 \pi)}|f(t)|<+\infty \quad$ for $p=+\infty$;

indeed, on the event $\Omega_{r, s, \delta_{0}}$, we check that $\left(\lambda \bar{d}_{\lambda}(t)-X_{t}\right)_{t \in(0,2 \pi)}$ is bounded uniformly on $(0,2 \pi)$ by $\lambda^{-1} s^{5} /\left(R_{\star}^{2} r^{2}\right)$ times an explicit constant depending only on $r, s$, and $\delta_{0}$. Hence, we obtain the following proposition.

Proposition 3. Almost surely, we have, for all $p \in[1,+\infty]$,

$$
\left(\lambda \bar{d}_{\lambda}(t)\right)_{t \in(0,2 \pi)} \stackrel{L^{p}}{\longrightarrow}\left(X_{t}\right)_{t \in(0,2 \pi)} \text { as } \lambda \rightarrow+\infty .
$$

\subsection{Estimate on the accuracy of the approximate defect process}

Obviously, the difference between the approximation $\left(\lambda \bar{d}_{\lambda}(t)\right)_{t \in[0,2 \pi]}$ and the rescaled defect process $\left(\lambda d_{\lambda}(t)\right)_{t \in[0,2 \pi]}$ is not convergent to 0 in the space $L^{\infty}(0,2 \pi)$ because of the (common) jumps of both the rescaled and limit processes. We prove the following result.

Theorem 8. Almost surely, we have the following convergence:

$$
\lim _{\lambda \rightarrow+\infty} \lambda \int_{(0,2 \pi)}\left|d_{\lambda}(t)-\bar{d}_{\lambda}(t)\right| \mathrm{d} t=0 .
$$

Proof. The proof of this theorem will also be carried out in two steps.

Step 1. Obtain estimates on the widths of the 'almost jumps' of the defect process on almost-full probability events.

Step 2. Obtain $L^{\infty}$ estimates on the difference of the two processes on the above events.

Step 1: Widths of the jumps. The quantities $d_{\lambda}$ and $\bar{d}_{\lambda}$ are distinct only in the following case: the first intersecting line in direction $t$ is not associated to the same point of the underlying marked Poisson point process $\boldsymbol{Z}$ as the first intersecting disc in direction $t$. This case decomposes into three subcases:

- the first intersecting disc is associated to another line of the line process that contains an edge of the Crofton cell, adjacent to the actual edge intersected by $\Delta_{t}$;

- the disc is associated to a nonadjacent edge;

- the disc is associated to a line that does not intersect the Crofton cell.

We shall show that the last two subcases can be excluded on some event. Let us first define, for $\varepsilon>0$, the thick Crofton cell as $\mathcal{C}_{\varepsilon}=\mathcal{C} \oplus B(0, \varepsilon)$. We shall say that it is equivalent to $\mathcal{C}$ if it has the same edges and vertices as $\mathcal{C}$; more precisely, if, for each $i \in\left\{1, \ldots, N_{e}\right\}$, we have

$$
\left(D_{i} \oplus B(0, \varepsilon) \cap D_{i+1} \oplus B(0, \varepsilon)\right) \cap\left(D_{i+1} \oplus B(0, \varepsilon) \cap D_{i+2} \oplus B(0, \varepsilon)\right)=\varnothing,
$$

where the $D_{i}, i \in\left\{1, \ldots, N_{e}\right\}$, are the lines supporting the edges of $\mathcal{C}$. The intersections $D_{i} \oplus B(0, \varepsilon) \cap D_{i+1} \oplus B(0, \varepsilon)$ are lozenges, denoted by $C_{i, \varepsilon}$ (crossings). 
Remark 4. It is clear that, by thickening the Poisson line process, the thick Crofton cell is defined by at most the lines on the boundary of the Crofton cell. Our notion of equivalence is a little more demanding than just assuming that all these lines bound $\mathcal{C}_{\varepsilon}$.

Lemma 4. Let $r<s$ and $\varepsilon$ be positive numbers, and define $\bar{\Omega}_{r, s, \varepsilon}$ to be the event such that, for $\omega \in \bar{\Omega}_{r, s, \varepsilon}$, we have the following hypotheses.

Hypothesis 1. The Crofton cell $\mathcal{C}(\omega)$ is included in $B(0, s)$ and contains $B(0, r)$.

Hypothesis 2. The thick Crofton cell $\mathcal{C}_{\varepsilon}(\omega)$ is equivalent to the Crofton cell $\mathcal{C}$, and, for each $(\rho, \theta, R) \in \boldsymbol{Z} \backslash\left\{\left(\Upsilon_{i}, \Theta_{i}, R_{i}\right), i \in\left\{1, \ldots, N_{e}\right\}\right\}$, we have

$$
\mathcal{C}_{\varepsilon}(\omega) \cap\left(D_{\rho, \theta} \oplus B(0, \varepsilon)\right)=\varnothing,
$$

and, for all $i \in\left\{1, \ldots, N_{e}\right\}$,

$$
C_{i, \varepsilon} \cap\left(D_{\rho, \theta} \oplus B(0, \varepsilon)\right)=\varnothing .
$$

Then we have

$$
\lim _{r, \varepsilon \rightarrow 0, s \rightarrow+\infty} \mathrm{P}\left(\bar{\Omega}_{r, s, \varepsilon}\right)=1 .
$$

Furthermore, for large enough $\lambda$ (depending on $r, s$, and $\varepsilon$, and $\left(\Upsilon_{i}, \Theta_{i}\right), i \in\left\{1, \ldots, N_{e}\right\}$ ) and all $t \in[0,2 \pi)$, the first intersecting line and disc are associated either to the same point $\left(\Upsilon_{i}, \Theta_{i}, R_{i}\right) \in Z$ or to the two points $\left(\Upsilon_{i}, \Theta_{i}, R_{i}\right)$ and $\left(\Upsilon_{i \pm 1}, \Theta_{i \pm 1}, R_{i \pm 1}\right)$, where $i \in$ $\left\{1, \ldots, N_{e}\right\}$ is the index of the corresponding edge of the Crofton cell.

The proof of this lemma follows classical lines; for instance, for fixed $r$ and $s$, it is obvious that the conditional probability, given that $R_{M} \leq s$ and $R_{m} \geq r$, that some line does violate (hypothesis 2) is of order $\varepsilon s$. The last point is clearly illustrated in Figure 7.

If $\lambda$ is chosen large enough, depending only on $r, s, \varepsilon$, and $\left(\Upsilon_{i}, \Theta_{i}\right)_{i \in\left\{1, \ldots, N_{e}\right\}}$, then it is clear, thanks to Proposition 2, that the angles $t$ for which the two edges are needed to determine the defect at $t$ are at most those corresponding to the disjoint sub-lozenges $C_{i, \eta / \lambda}$ formed by the intersections of two polar thick lines $D_{i} \oplus B(0, \eta / \lambda)$ and $D_{i+1} \oplus B(0, \eta / \lambda)$ corresponding to edges $i$ and $i+1$, where $\eta$ depends on $r, s, \varepsilon$, and $\left(\Upsilon_{i}, \Theta_{i}\right)_{i \in\left\{1, \ldots, N_{e}\right\}}$, with $\eta / \lambda<\varepsilon$.

This implies that the total length of problematic angles $t$ is less than $N_{e} \zeta / \lambda$, where $\zeta$ depends in quite a technical way on $r, s, \varepsilon$, and $\mathcal{C}$ through the minimum of the differences $\left|\Theta_{i+1}-\Theta_{i}\right|$.

Step 2: $L^{\infty}$ bounds. From the properties of the Crofton cell on the set $\bar{\Omega}_{r, s, \varepsilon}$, we may easily evaluate exactly the difference between both processes; indeed, when this difference is nonzero,

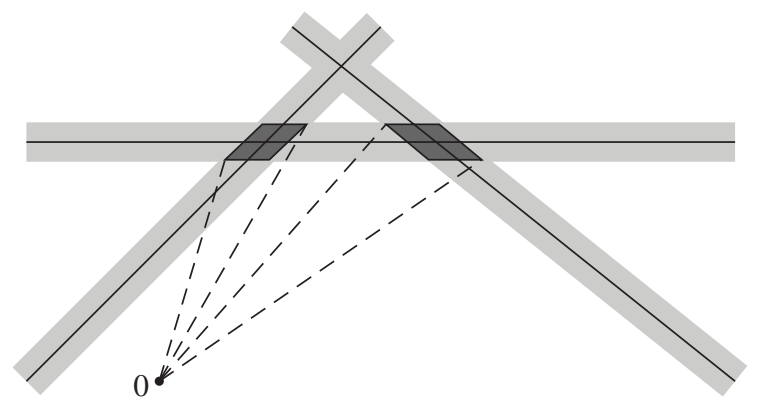

FIGURE 7: Thick Crofton cell and lozenges. 
it is less than

$$
\left|d_{\lambda}(t)-\bar{d}_{\lambda}(t)\right| \leq d_{1}(t)+d_{2}(t)+d_{3}(t),
$$

where $d_{1}(t)$ is the modulus of the distance between the intersections on $\Delta_{t}$ of $D_{i}$ and the associated disc $B_{i}, d_{2}(t)$ is the modulus of the distance between the intersections on $\Delta_{t}$ of $D_{i \pm 1}$ and the associated disc $B_{i \pm 1}$, and $d_{3}(t)$ is the modulus of the distance between $D_{i} \cap \Delta_{t}$ and $D_{i \pm 1} \cap \Delta_{t}$ (see Figure 7).

Let us note that one of $d_{1}(t)$ and $d_{2}(t)$ is exactly $\bar{d}_{\lambda}(t)$; thanks to Proposition 2, these two terms are bounded from above by a constant for large enough $\lambda$ :

$$
d_{j}(t) \leq \frac{1}{\lambda R_{*}} \frac{s}{r}\left(s+\frac{s^{2}}{\lambda R_{*}}\right)^{2}, \quad j=1,2 .
$$

The third term is also bounded from above by a constant $M(r, s, \varepsilon, \mathcal{C}) \times \eta / \lambda$. Hence, we obtain

$$
\lambda\left|d_{\lambda}(t)-\bar{d}_{\lambda}(t)\right| \leq M_{1}(r, s, \varepsilon, \mathcal{C}) .
$$

Hence, we have the following estimate on the $L^{1}$-norm of the difference:

$$
\lambda \int_{(0,2 \pi)}\left|d_{\lambda}(t)-\bar{d}_{\lambda}(t)\right| \mathrm{d} t \leq \frac{\zeta N_{e}}{\lambda} M_{1}(r, s, \varepsilon, \mathcal{C}) .
$$

This upper bound converges towards 0 for each $\omega$ in the set $\bar{\Omega}_{r, s, \varepsilon}$. This concludes the proof of Theorem 8 .

As a consequence of this result and of Proposition 3, we eventually obtain the following convergence.

Theorem 9. Almost surely, we have

$$
\left(\lambda d_{\lambda}(t)\right)_{t \in(0,2 \pi)} \stackrel{L^{1}(0,2 \pi)}{\longrightarrow}\left(X_{t}\right)_{t \in(0,2 \pi)} \text { as } \lambda \rightarrow+\infty
$$

where the process $\left(X_{t}\right)_{t \in(0,2 \pi)}$ is defined in Theorem 7 .

\subsection{Tail probability for the supremum of the defect process}

This short section is devoted to a uniform bound on the tail probabilities for the defect processes for large $\lambda \mathrm{s}$.

Proposition 4. We have the following estimate: for all $\beta<1$,

$$
\lim _{u \rightarrow+\infty}\left\{s^{\beta} \limsup _{\lambda \rightarrow+\infty} \mathrm{P}\left(\sup _{\theta \in[0,2 \pi)} \lambda\left|d_{\lambda}(\theta)\right| \geq u\right)\right\}=0
$$

Sketch of the proof. The proof uses the same tools as before: the estimates on the growth of both $\mathcal{C}$ and the empty component of the Boolean model around the origin. Indeed, we may give an explicit upper bound of the defect on the set $\bar{\Omega}_{r, s, \varepsilon}$ using the computations of Section 5.2. This bound is roughly of order $s^{5} / r^{2}$. By using an adequate choice of $r \rightarrow 0$ and $s \rightarrow+\infty$ in terms of powers of $u$, we obtain the result. This tedious proof is left to the interested reader. 
Remark 5. (Splitting the defect process.) The limit defect process may be decomposed into a continuous part and a pure-jump part. Such a splitting can be done for the defect process at fixed $\lambda$; indeed, let us write

$$
\lambda d_{\lambda}(t)=\lambda \overline{\bar{d}}_{\lambda}(t)+\left(\lambda d_{\lambda}(t)-\lambda \overline{\bar{d}}_{\lambda}(t)\right)
$$

where $\overline{\bar{d}}_{\lambda}$ is the continuous part of the process $\bar{d}_{\lambda}$ (this is almost surely defined as being equal to $\bar{d}_{\lambda}$ at the angle $t=0$, and the jumps are deleted). Then it can be shown that both terms above converge in law: the first term converges in law in $C([0,2 \pi])$, the space of continuous functions defined on $[0,2 \pi]$, and the second term converges in law in a weak sense.

Remark 6. (Directions for the general case.) For more general shapes, the coupling is more tricky to obtain. We may proceed in the following way.

- Start with a homogeneous Poisson line process.

- Mark this process with smooth convex shapes with no flat portion on the boundary: $\tau(F)$, where $\tau$ is a uniform rotation and $F$ is a smooth convex random closed set.

- Given $\tau, F$, and the Poisson line, consider the centre of the rotated shape (rescaled by a factor $\lambda$ ) tangent at the line, at the same distance from the origin as the line.

- Consider the point process of those centres of shapes and modify it so that its intensity measure becomes the Lebesgue measure multiplied by the parameter $\lambda$. This will be done by a function

$$
(\rho, \theta, \tau, F) \mapsto \Psi_{\lambda}(\rho, \theta, \tau, F) \in \mathbb{R}_{+} \times[0,2 \pi) .
$$

Using this procedure, the computations might involve more technical details, for example, the limiting process might be expressed with the curvature of $F$.

\section{Acknowledgement}

The authors wish to thank the anonymous referee for numerous remarks that greatly helped to improve the first version of this paper.

\section{References}

[1] Billingsley, P. (1999). Convergence of Probability Measures, 2nd edn. John Wiley, New York.

[2] Calka, P. (2002). The distributions of the smallest disks containing the Poisson-Voronoi typical cell and the Crofton cell in the plane. Adv. Appl. Prob. 34, 702-717.

[3] Calka, P. (2003). Precise formulae for the distributions of the principal geometric characteristics of the typical cells of a two-dimensional Poisson-Voronoi tessellation and a Poisson line process. Adv. Appl. Prob. 35, 551-562.

[4] Hall, P. (1985). Distribution of size, structure and number of vacant regions in a high-intensity mosaic. Z. Wahrscheinlichkeitsth. 70, 237-261.

[5] Hall, P. (1988). Introduction to the Theory of Coverage Processes. John Wiley, New York.

[6] Heinrich, L., Schmidt, H. And Schmidt, V. (2006). Central limit theorems for Poisson hyperplane tessellations. Ann. Appl. Prob. 16, 919-950.

[7] Hug, D., Reitzner, M. And Schneider, R. (2004). The limit shape of the zero cell in a stationary Poisson hyperplane tessellation. Ann. Prob. 32, 1140-1167.

[8] Matheron, G. (1975). Random Sets and Integral Geometry. John Wiley, New York.

[9] Michel, J. And Paroux, K. (2003). Local convergence of the Boolean shell model towards the thick Poisson hyperplane process in the Euclidean space. Adv. Appl. Prob. 35, 354-361.

[10] Miles, R. E. (1964). Random polygons determined by random lines in a plane. Proc. Nat. Acad. Sci. USA 52, 901-907. 
[11] Miles, R. E. (1964). Random polygons determined by random lines in a plane. Proc. Nat. Acad. Sci. USA 52, $1157-1160$.

[12] Molchanov, I. S. (1996). A limit theorem for scaled vacancies of the Boolean model. Stoch. Stoch. Reports 58, 45-65.

[13] Molchanov, I. (2005). Theory of Random Sets. Springer, London.

[14] Paroux, K. (1997). Théorèmes centraux limites pour les processus poissoniens de droites dans le plan et questions de convergence pour le modèle booléen de l'espace euclidien. Doctoral Thesis. Université Lyon 1.

[15] PAroux, K. (1998). Quelques théorèmes centraux limites pour les processus Poissoniens de droites dans le plan. Adv. Appl. Prob. 30, 640-656.

[16] SchneIder, R. (1993). Convex Bodies: the Brunn-Minkowski Theory (Encyclopedia Math. Appl. 44). Cambridge University Press.

[17] Stoyan, D., Kendall, W. S. And Mecke, J. (1995). Stochastic Geometry and Its Applications, 2nd edn. John Wiley, Chichester. 